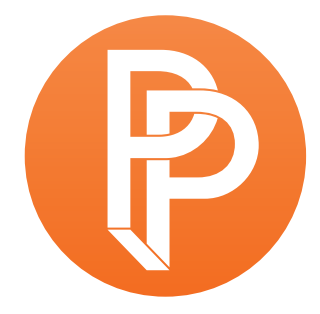

PERFORMANCE

PHILOSOPHY

\title{
DANCE, PHILOSOPHY, AND SOMAESTHETICS
}

\section{ERIC MULLIS QUEENS UNIVERSITY OF CHARLOTTE}

\begin{abstract}
Philosophy
Anyone who discusses the possibility of an artistic medium doing philosophy must begin by saying something about what they take philosophy to be. Drawing on a recent debate in philosophy of film, two central positions on the issue can be outlined (Wartenberg 2006). One emphasizes that philosophy is an academic discipline that features the articulation and debate of arguments on specific conceptual issues. If a film or a dance cannot advance arguments on an issue then, on this account, they cannot do philosophy. A more expansive understanding of philosophy, however, holds that a philosopher need not develop a specific argument-complete with a thesis, supporting evidence, and conclusion-but may provide significant insight and stimulate reflection on conceptual issues. Martin Heidegger's work falls within this category as it provides a great deal of insight into the human existential condition which in turn encourages the reader to consider his or her own experience. Similarly, a film or dance may provide insight on philosophical concepts by embodying them and possibly by pushing them beyond their original formulation in a way that fosters reflection on the part of the viewer. For example, William Forsythe's dance installation Human Writes (2012) uniquely performs the tension that exists between a political ideal and the practical difficulties that arise when trying to universalize it. It also fosters criticism of the inconsistent manner in which industrialized nations, often because of economic or political reasons, do not take enough steps to ensure that the basic human rights of many who live in developing countries are sufficiently protected. In this way, Human Writes embodies a key idea from contemporary political philosophy and encourages audience members to critically assess that ideal.
\end{abstract}


However, I would like to shift attention away from the philosophical import of completed dance works such as Human Writes and focus on dance processes that are often used to develop them in the studio. From this perspective the relationship has less to do with the philosophical contentsuch as universal human rights - that dances may advance and more to do with creative dance processes that can be used advance philosophical inquiry into the nature of human embodiment. In the remainder of this essay I will argue that the dancer is doing philosophy when he or she is using dance technique to consciously foster new somaesthetic experience and to actively investigate the manner in which embodied experience is influenced by particular social values. The first component of my argument draws on pragmatist philosophy and more specifically on the work of Richard Shusterman, while the second draws on concepts advanced by Michel Foucault and Susan Bordo.

With regard to methodology, dance philosophy can emphasize the first-hand experience of making and performing dances and it can, in a more analytical fashion, engage with conceptual issues raised by those practices. The philosophical approach that I use to investigate Gaga and Contact Improvisation is pragmatist in that it emphasizes the manner in which the experiential and the reflective phases of experience mutually inform one another. One could take a more analytical approach to the dance techniques discussed here (for example, see McFee 1992; Bunker et al. 2013) or a more continental approach (for example, see Hall 2012; LaMothe 2015; McCormack 2014) and since I believe that discussions informed by those approaches would reveal important insights, I will include references to relevant literature through the course of the essay.

Regardless of methodological commitments, I believe that it is important for dance philosophers to be quite specific about the dance practices that they are considering. Nietzsche famously suggested that philosophers can be dancers but it is quite unclear what he took dancing to be ([1883] 1978, 107-110). Was he thinking of vernacular forms such as folk dance? Or dance for the concert stage such as pantomime or classical ballet? More generally, was he thinking of choreographed or improvised dance? These questions are largely rhetorical but they point to the fact that historical and contemporary philosophers often refer quite generally to dancing because they have rudimentary understandings of the wide range of dance practices characteristic of dance education, choreography, and performance. But if we are going to consider the manner in which dance qua dance can do philosophy, then generalities must be avoided and the philosophical import of specific dance practices considered. For this reason, this essay considers the intersection of two specific dance improvisation techniques and self-reflective embodied research and it primarily references the work of dance scholars who have first-hand experience with dance practice.

\section{Somaesthetic Inquiry}

Drawing on the pragmatist philosophy of John Dewey, Richard Shusterman developed the field of somaesthetics which theoretically and practically investigates the manner in which the body functions as a source of aesthetic experience (Shusterman 2000, 262-283). Throughout his career, Dewey consistently argued against mind-body dualism and emphasized that individuals can grow 
to the extent that they can develop intelligent physical and mental habits that mediate the problematic situations that arise in daily life ([1925] 1958). Further, in his later work he emphasized that aesthetic experience can arise in a range of day-to-day activities and is not restricted to encounters with fine art housed in galleries and museums. On his account, fine arts such as dance clarify and intensify the energies that culminate in aesthetic experience in everyday life such as when we cook a meal, exercise, have a robust conversation with friends, enjoy a soccer game, or hike a mountain trail (Alexander 1987, Dewey [1932] 2005). Shusterman develops this approach by considering the manner in which a range of body practices from different cultural traditions can be practiced in order to consciously create rich and varied somaesthetic experience for practitioners (2010, 262-338). This meliorist approach emphasizes that bodily experience is not a given but can be intentionally developed such that new aesthetic experiences can be cultivated and appreciated by the individual. How does dance practice then, fall within this program?

Dance is like yoga, tai chi chuan, martial arts, running, and sport in that it provides a systematic framework that contextualizes a practitioner's cultivation of new somaesthetic experiences. Yoga, for example, includes a system of exercises that are designed to realize specific psycho-physical goals. The system allows one to experience novel proprioceptive sensations as one cultivates its specific poses, movement sequences, breathing techniques, and general meditative focus (Mullis 2015). The consequent increase in whole-body endurance strength, flexibility, and integration of mind, body, and breath allows the practitioner to aesthetically appreciate what may for them bodily experiences that may be indicative of a new mode of embodiment.

One may agree that this indeed is a first-hand embodied investigation into somaesthetic experience but disagree that it is properly philosophical in nature since it does not present or advance specific philosophical ideas in the manner described above. There are two ways to reply to this. The first is to point to eastern practices that manifest distinct philosophical traditions and their respective philosophies of embodiment. Modern Postural Yoga is historically connected with Ayurvedic medicine and a philosophy of embodiment that is conceptually intertwined with classical Hindu philosophy (Singleton 2010). Tai chi is based in concepts drawn from classical Daoist philosophy, most eastern martial traditions embody aspects of Confucian ethics, and many body practices that developed in Japan-such as the tea ceremony-physically express ideas central to Zen Buddhism (Schmeig 2004). But with this said, many practitioners of these body traditions do not place much stake on the philosophies that were seminal to their development and, further, there are many somaesthetic practices-such as sport, hiking, running, and eating-that do not manifest aspects of particular philosophical traditions.

The second and more substantial reply to the skeptic entails drawing attention to the manner in which a somaesthetic practice can be philosophical if it leads the practitioner to engage in selfreflection about general characteristics of his or her embodied experience. When this occurs, he or she begins to consider personal and cultural factors that fostered the development of his particular mode of embodiment which in turn allows him to consider alternatives. This is philosophical for three reasons. 
The first is that it entails a level of self-understanding consistent with the Socratic dictum to "know thyself." It will be remembered that Socrates had a high degree of self-knowledge concerning his lack of epistemic certainty and that the Platonic dialogues demonstrate that Socrates' interlocutors lacked this kind of self-knowledge as they unknowingly held many beliefs that could not withstand logical scrutiny. Although it has less to do with epistemic consistency, an important component of Shusterman's (2008) work concerns the manner in which a range of more contemporary philosophers had troubling relationships with their own bodies, relationships that often unknowingly influenced their philosophies of embodiment. Shusterman's discussion of the relationship between the biographies of philosophers such as William James, Ludwig Wittgenstein, Simone De Beauvoir, and Maurice Merleau-Ponty and their respective understandings of embodiment provides a critical foundation for an approach that does not view embodied experience as a given. More specifically, this aspect of Shusterman's work demonstrates that the understandings of the body articulated by these philosophers were unknowingly inflected by a range of specific cultural values that they took as given and this observation in turn encourages readers to self-reflectively consider the manner in which personal experience within a particular cultural context has shaped their own experience of embodiment.

This critical self-reflection leads us to the second reason why somaesthetics is philosophical in nature, namely, the manner in which critical somaesthetics opens to door to intelligent selffashioning outlined by John Dewey. Dewey ([1922] 2002) emphasized that experience becomes increasingly meaningful when the individual develops an array of mental and physical habits that allow her to pursue the experience of a plurality of value within an ever-changing physical and social environment. Once she considers the manner in which particular cultural values have shaped her mode of embodiment, she can then consider how different body practices can allow her to pursue increasingly rich somaesthetic experience. Dewey advocated for the work of Frederick Matthias Alexander whose Alexander Technique teaches individuals to become aware of unnecessary muscular tension and postural problems that occur while performing everyday activities (Dalton 2002, 82-86). Because of a history or repeated usage, the individual's perceptions of muscular tension, relaxation, and postural integrity may be skewed and the Alexander technique allows the practitioner to cultivate increasingly accurate physical perceptions. In turn, this makes possible somaesthetic experiences that previously lay outside of the practitioner's somatic framework. Dewey advocated for the Alexander technique because it practically demonstrated how habits can be rendered intelligible and how that process can foster the pursuit or a plurality of value that renders the individual's experience richer and more meaningful.

To recap the previous two points, somaesthetic practices can do philosophy if the individual who engages them develops a self-reflective inquiry into their embodied experience to the extent that it plays into a broader project of cultivating the experience of a plurality of value. Further, somaesthetics is philosophical in nature because it fosters an embodied understanding of the general manner in which the body structures human experience. Through embodied practice and reflection I learn about the possibilities of somaesthetic experience for myself and this leads me to consider the experience of those who engage in body practices that I have little experience with and perhaps find quite odd. I learn of the wide range of embodied experience which in turn 
encourages me to consider the general plasticity of the human body, the nature and function of habit, and the relationship between the body and the self. If the preceding points are correct, somaesthetics does philosophy when it advances an active experiential research into human embodiment. But again, what does this have to do with dance?

If we return to Dewey's point about the relationship between fine art and aesthetic experience in everyday life, we find that dance as a fine art refines and expresses energies that arise in the course of daily life. This occurs because dancers generally master expressive dance technique that allows them to clarify the movements of the body, to articulately express somatic energy into performance spaces, and to develop a performative presence that increases the significance of their postures and actions. In the studio, the end of any particular action is ultimately artistic expression whereas in day to day life the end of an action is contextualized by specific projects such as getting to work, finishing an assignment, teaching class, and so on. As discussed, dance is like other body practices that provide practical and theoretical frameworks that can ground the cultivation of rich somaesthetic experience, however, it is unique in that the dancer's mode of embodiment is developed in the studio so that it ultimately may realize the artistic ends. For this reason, the techniques utilized by dancers remain distinct from those of other body practices since such techniques are intertwined with the process of variously giving form to artistic content. In order to make this point clearer, I will go beyond generalities and discuss two improvisation techniques that I have studied in the studio and that can be utilized by dance artists to generate aesthetically expressive movement.

\section{Improvisational Technique}

\section{Gaga}

Gaga technique was developed by the current director of the Batsheva Dance Company, Ohad Naharin who studied ballet and modern dance early in his dance career. In the early 2000s he developed a systematic approach to dance improvisation that can be practiced by dancers and non-dancers alike (Aldor 2003, 92-94). Gaga classes are divided into two categories: "Gaga Dancers" which offers professional dancers the opportunity to incorporate Gaga movement principles into their existing dance practice and "Gaga People" which can be practiced by anyone interested in investigating the possibilities of expressive movement. Gaga technique has several components but I will focus on two that are common to both Gaga Dancers and Gaga People and which are most pertinent for this essay: the kinesthetic pleasure of improvised organic movement and the phenomenological investigation of somatic textures.

Gaga is an improvisation technique that emphasizes un-planned movement instead of pre-set choreographic patterns and sequences. Sessions generally begin with "floating" in which the practitioner imagines that the body is surrounded by water. This simultaneously produces a sense of physical support and gentle sense of ease in the body which the instructor develops by encouraging students to explore the manner in which imagined currents of water can move specific parts of the body and, via kinetic chains, affect the body as a whole. In this way the imagination is used to create a mode of embodiment that is characterized by flowing, integrated 
movement. This leads Naharin to emphasize that Gaga includes a therapeutic component in that it entails accepting the body as is instead of molding it into codified technique that is designed to realize particular choreographic ends (2006: 53).

In daily life we generally experience somatic pleasure as we engage in physical practices with particular goals in mind. However, the goal of floating is to simply enjoy moving in an imaginative and exploratory fashion which-not unlike other dance improvisation techniques-fosters a nonnarrowly instrumental relationship to the body. The dancer's attention is attuned on the phenomenological experience of the movement because her imagination actively contextualizes the movement and because she experiments with non-habitual movements that require more attention to execute (for phenomenological approaches to dance movement, see Fraleigh 1996; Sheets-Johnstone 2015).

A second and related aspect of Gaga technique is the cultivation of the experience of a range of somatic textures. In daily life the musculature of the body generally manifests limited degrees of tension and relaxation and Gaga asks practitioners to use their imaginations to intentionally experience a broader range of somatic texture. Floating fosters a sense of somatic liquidity and this can be developed further by imagining that specific surfaces of the body are hard as armor, that they are elastic in the manner of stretchy materials with different degrees of elasticity, or that the limbs are masses of concrete. Further, one can explore variations in the transitions from one texture to another as well as variations in the rate of transition. Time is spent investigating how different somatic textures somaesthetically reveal somatic surface and depth and how they more generally affect improvised movement quality (for more on the phenomenological experience of surface and depth in dance, see Kozel 2007, 28-40).

I have been focusing on two components of Gaga technique that can be practiced by dancers and non-dancers alike. Professional dancers can use floating and somatic textures purely for movement exploration purposes but in addition these strategies can be used to create choreography that can be incorporated into dance pieces. Indeed, in many of the pieces that are included in Batsheva's repertory, one finds dancers floating and manifesting dramatic changes in somatic textures. This and other components of the approach produce a distinctive somaesthetic style that assists in the expression of Naharin's choreographic sequences. Regardless, I would like to emphasize that the dancer or non-dancer who invests in Gaga technique is executing an experiential philosophical investigation of embodiment as described in the previous section.

Instead of taking the experience of embodiment as a given, the improvisation technique allows the dancer to imaginatively experiment with movements that create new somaesthetic experiences. Gaga reveals that the investigation can be therapeutic in nature and it demonstrates that it can be developed in order to create choreography for the concert stage.

\section{Contact Improvisation}

I would like to consider another improvisation technique that was developed in the 1970s by Steve Paxton and that can be used for experiential or performative ends-Contact Improvisation (CI). 
Whereas Gaga focuses primarily on one's internal somaesthetic experience, Cl necessarily involves a partner and thereby requires one to focus on physical interactions with another.

As the name implies, $\mathrm{Cl}$ generally emphasizes remaining in contact with one's partner as various surfaces of the body are used to articulate spontaneous and cooperative movement sequences. Partners must continually sense changing points of physical strength and weakness (both in their own bodies and in that of their partner's), sense the flow of kinetic energy, and remain aware of movement pathways that may be utilized in an efficient channeling of that energy. Further, since movement is improvised and since verbal communication is generally discouraged, each partner must remain sensitive to the movement-intentions of the other so that the dance can unfold in a mutually cooperative manner (Novack 1990).

$\mathrm{Cl}$ is akin to Gaga technique in that it can be practiced by dancers and non-dancers and because it is as an improvisational dance process that provides a structure for the cultivation of somaesthetic experience in the studio that can be developed into choreography for the stage. However, since $\mathrm{Cl}$ entails working in close contact with a dance partner, the embodied investigation that it can advance differs from that of Gaga practice. Specifically, $\mathrm{Cl}$ offers an experiential investigation into the manner in which external physical forces can affect the moving body, into the manner in which the phenomenological boundary between self and other can be blurred, and into the manner in which cultural values inflect the experience of touch.

Paxton referred to the practice as an experiential investigation into the kinetic physics of the human body $(1975,41)$. In solo practice, one can develop an appreciation of the structural integrity of one's frame in relation to the ground and to the force of gravity and in $\mathrm{Cl}$ this appreciation is heightened since one's physical structure unfolds in relation to a partner's moving body. That is, human movement is always a functional negotiation of the human frame and its musculature with the force of gravity and this negotiation is intensified when one begins to improvise in close contact with another body. The negotiation is triangulated as I interact with the force of gravity and interact with physical forces that characterize my partner's movement intentions. This continually changing interaction encourages me to focus on the present moment and to become aware of the manner in which the kinetic capacities of our bodies can cooperatively relate to gravity and the ground. Here again we find a dance practice that experientially investigates a fundamental aspect of human embodiment. Whereas Gaga allows the practitioner the chance to use the imagination to develop somatic textures in solo practice, $\mathrm{Cl}$ allows her to investigate the structural integrity of the human frame as it interacts with that of another.

While performing $\mathrm{Cl}$ movement research the individual can enter into a flow state in which the boundary between the self and the other becomes less well defined (for more on this with regard to dance, see Fraleigh 1996, 57-70; Kozel 2007, 136-160). There are several factors that play into this experience. The first concerns the necessity of ongoing focused attention on improvised bodily experience in the present moment. If one's reflective mind takes one out of the experience of the present, $\mathrm{Cl}$ provides immediate feedback since one will no longer be pre-reflectively responding the partner's movement-intentions. Reflection results in inappropriate physical 
responses, missed opportunities for execution of efficient movements, and more generally a disruption of the kinetic flow of the interaction. As with yoga and tai chi, a meditative focus on the body allows the preoccupations of the reflective mind to fall by the wayside and consequently allows one to stay present to the continually changing characteristics of the interaction. $\mathrm{Cl}$ is unique, however, in that close physical proximity contributes to the blurring of the boundary between self and other. At a distance, my partner is a distinct embodied individual but as our bodies cooperatively move in contact we can experience moments in which intentions are effortlessly realized and the distinction between the doer and what is done momentarily dissolves. In this way $\mathrm{Cl}$ provides the context for a unique experiential investigation into the possibilities of the self in physical activity (Cooper Albright 2011, 9-16).

There are many factors that are necessary for the experience of improvised physical effortlessness with a partner. Both partners must have a certain level of physical ability, must be sensitive to the movement-intentions of the other (e.g. must be continually "listening"), and must be attuned to the possibilities of the continually changing kinetic architecture that unfolds in relation to gravity and the floor. Importantly, there must be a high degree of trust between the two partners as physical safety is always an issue. Whenever I press weight into my partner I must trust that she will react in a way that counters my unstable and physically vulnerable position; counteracting it by pressing weight into me or by essentially catching me. Further, since $\mathrm{Cl}$ is an improvisation technique my shift of weight may very well be unexpected and I must trust that she is continually sensitive to my actions.

A key component of $\mathrm{Cl}$ is touch and in any culture there are norms surrounding acceptable touch, norms that center on social differences such as age, gender, sexuality, and social position. Further, social context invariably inflects such norms. Touch is generally minimized in professional contexts but often expected when intimacy is expressed in the privacy of the home. There is much to say here but, for the sake of brevity, I will focus on social customs of touch that pertain to the male gender.

Briefly, western cultures are characterized by specific norms concerning the manner in which men touch other men (Bordo 2000, 50-64). When meeting an adult male for the first time one shakes his hand and, once a relational bond has been formed, possibly embraces him at particular times (e.g. during a celebration, bidding farewell, etc.). Men can make physical contact within the context of physical competition as well. They strike each other in different ways as they tackle or block each other or as they celebrate a successful play. These examples demonstrate that contact between men generally has clear duration and degree of force. A handshake or embrace should not last too long and contact should be characterized by firmness.

Steve Paxton's first explorations of $\mathrm{Cl}$ were developed with male college athletes and the partnering was aggressively kinetic. However, in time, $\mathrm{Cl}$ practitioners began to emphasize more effortless interactions which require a high level of physical relaxation, perceptual sensitivity, and a more generous touch in which any surface of one's body can sensitively remain in contact with any surface of a partner's body. This kind of touch may not be problematic for dancers who have 
a wealth of experience with dance partnering but it can be uncomfortable for non-dancers whose bodies have been, often unknowingly, inscribed by standards of appropriateness that centre on touch. Specifically, it allows practitioners to consider how their sense of appropriate touch manifests values concerning gender and gender relationships which are the product of a process of enculturation that begins in early childhood and that is developed in a range of cultural institutions and settings across one's life. In this way $\mathrm{Cl}$ offers a physical investigation into Foucault's account of bio-power, that is, into the manner in which social values are, via habit, inscribed in the body and often unconsciously manifested in bodily attitudes and actions (2001). Though he does not discuss dance, Michel Foucault's notion of "docile bodies" discussed in Discipline and Punish can be seen in the case of dance students. Foucault discusses how the social power that characterizes particular social institutions such as the prison, school, or army barracks is internalized by the obedient bodies of inmates, students, and soldiers and dance scholars have noted that this also often pertains to students in the dance studio (Alterowitz 2014; Green 2003). Whereas codified dance technique taught in dance schools requires regimentation, examination, and clear social roles, $\mathrm{Cl}$ demonstrates how a more collaborative improvisational dance practice can foster awareness of bio-power and, following Albright (2011), can provide a site of resistance to the techniques that render the body obedient to dominant social values (for more on dance practice and social resistance, see Albright 1997; Hall 2011; Summers-Bremner 2009). In practicing $\mathrm{Cl}$, I can learn how pre-reflective bodily attitudes inflect the experience of touch and can practice developing new attitudes that are based not in dominant social values but in intentional experimental practice.

I have considered how $\mathrm{Cl}$ can lead one to self-reflectively consider the manner in which social norms foster a sense of appropriateness concerning embodiment, touch, and gender. In addition, the experience of physically cooperative trust characteristic of $\mathrm{Cl}$ grounds embodied investigation into ethical attitudes toward others. Shusterman observes that bodily awareness can reveal ethical attitudes to others that are inconsistent with egalitarian values (2008: 5-7). I may believe that, on principle, all human beings deserve equal ethical respect but come to realize that my embodied reactions to perceptions of otherness-whether rooted in gender, ethnicity, or sexuality-betray that belief. I may notice feelings of physical discomfort, anxiety, or disgust arise as I encounter certain others thereby creating a dissonance between what I cognitively and physically believe.

Iris Young (1990) and Susan Bordo's (2004) work draws attention to social standards concerning gender, body morphology, and ability, standards which have implications in daily life, in the dance studio, and on the concert stage. Those standards are relevant for the practice of $\mathrm{Cl}$ since physical trust, for example, can be undermined by the internalized association of female gender and physical passivity. Further, such standards are often codified in dance traditions that outline norms of partnering in which women are displayed and physically lifted by their male counterparts (Foster 1998). Consequently, a woman new to Cl may not believe that she has the physical ability to support the weight of a partner and she may, because of an internalized association of the feminine with passivity, believe that it is inappropriate for women to actively partner another. Conversely, a male dancer may not be willing to trust a female $\mathrm{Cl}$ partner by giving her his weight and may believe that it is inappropriate for him to be lifted. 
$\mathrm{Cl}$ provides a setting and techniques for experimentation with the capacities of bodily structure, a site of resistance to social standards concerning gender, and thereby allows for the gradual development of trust. The dancers can learn of the manner in which the structural integrity of the body paired and proper movement pathways can-regardless of gender-support a great deal of weight. In turn, this process can alert practitioners to habitual embodied reactions to perceptions of otherness that often belie ethical convictions and can foster change thereby contributing to a more robust egalitarian ethic.

I have drawn on contemporary feminist philosophy in order to focus on social values and ethical attitudes associated with gender and ability, however, since $\mathrm{Cl}$ is a physically cooperative activity that involves touch and the cultivation of trust, it can similarly be used to cultivate an embodied ethic that counters the tendency to react to perceptions of otherness. Regardless of my partner's ethnicity, sexuality, or political ideology, we can work to create the physical dynamic necessary for efficient partnering and in this way $\mathrm{Cl}$ enacts an embodied ethics.

\section{Conclusion}

This article demonstrates that Gaga and $\mathrm{Cl}$ do philosophy as they enact experiential and selfreflective investigations into particular aspects of human embodiment. Dance artists naturally strive to investigate what the medium of their art form can do since different modes of embodiment can translate into new approaches to choreography and dance aesthetics. In this case, intentional embodied inquiry can procure new somaesthetic experience, foster awareness of social values that can inflect the experience of others, and foster an embodied ethics. Since I have first-hand experience with the practices, I focused on Gaga Technique and $\mathrm{Cl}$, however, other improvisation techniques and choreographic processes used in the studio will ground other lines of embodied philosophical inquiry as will performance practices. This philosophy of practice demonstrates that robust dance philosophy is inter-disciplinary in that it entails in-depth and detailed investigation into the wide range of activities developed by dance artists.

\section{Note}

An earlier draft of this essay was presented at the 2014 annual meeting of the American Society for Aesthetics. I would like to thank members of the panel for their comments and suggestions: Mark Franko, Anna Pakes, and Aili Bresnahan. I would also like to thank the anonymous referees of this journal for their thoughtful comments and suggestions on an earlier draft of this essay. I would also like to thank dance instructors at American Dance Festival (Jesse Zaritt and Curt Haworth) and at the University of Wisconsin (Maria Gillespie and Daniel Burkholder) for their stimulating reflections on improvised dance. 


\section{Works Cited}

Albright, Ann Cooper. 2011. "Situated Dancing: Notes from Three Decades in Contact with Phenomenology." Dance Research Journal 43 (2): 7-18. http://dx.doi.org/10.1017/50149767711000027

_-_. 1997. Choreographing Difference: The Body and Identity in Contemporary Dance. Middletown: Wesleyan University Press.

Alexander, Thomas. 1987. John Dewey's Theory of Art, Experience and Nature: The Horizons of Feeling. New York: State University of New York Press.

Aldor, Gaby. 2003. "The Borders of Contemporary Israeli Dance: 'Invisible Unless in Final Pain."' Dance Research Journal 35 (1): 81-97. http://dx.doi.org/10.1017/S0149767700008780

Alterowitz, Gretchen. 2014. “Toward a Feminist Ballet Pedagogy: Teaching Strategies for Ballet Technique Classes in the Twenty-First Century." Journal of Dance Education 14 (1): 8-17. http://dx.doi.org10.1080/15290824.2013.824579

Bordo, Susan. 2004. Unbearable Weight: Feminism, Western Culture, and the Body. Oakland: University of California Press.

_- - 2000. The Male Body: A New Look at Men in Public and Private. New York: Farrar, Straus, and Giroux.

Bunker, Jenny and Anna Pakes, Bonnie Rowell, eds. 2015. Thinking Through Dance: The Philosophy of Dance Performance Practices Oxford: Dance Books.

Dalton, Thomas. 2002. Becoming John Dewey: Dilemmas of a Philosopher and Naturalist. Bloomington: Indiana University Press.

Dewey, John. (1932) 2005. Art as Experience. New York: Perigree.

. (1922) 2002. Human Nature and Conduct: An Introduction to Social Psychology. New York: Prometheus.

- (1925) 1958. Experience and Nature. New York: Dover.

Foster, Susan Leigh. 2011. Choreographing Empathy: Kinesthesia in Performance. New York: Routledge.

___. 1998. "Choreographies of Gender." Signs: Journal of Women in Culture and Society 24 (1): 1-33. http://dx.doi.org/10.1086/495316

Foucault, Michel. 2001. Power. Vol .3 of The Essential Works of Foucault 1954-1984, edited by James Faubion. Translated by Robert Hurley. New York: New Press.

Fraleigh, Sondra Horton. 1996. Dance and the Lived Body. Pittsburgh: University of Pittsburgh Press.

Green, Jill. 2003. "Foucault and the training of docile bodies in dance education." Journal of the Arts and Learning Special Interest Group of the American Education Research Association 19 (1): 99-125.

Hall, Joshua M. 2012. "Choreographing the Borderline: Dancing With Kristeva." Philosophy Today 56 (1): 49-58. http://dx.doi.org/10.5840/philtoday201256124

___. 2011. "Revalorized Black Embodiment: Dancing with Fanon." Journal of Black Studies 42 (2): 127-140. http://dx.doi.org/10.1177/0021934711419363

Nietzsche, Frederick. (1883) 1978. Thus Spoke Zarathustra, edited and translated by Walter Kaufmann. New York: Penguin.

Kozel, Susan. 2008. Closer: Performance, Technologies, Phenomenology. Cambridge: MIT Press.

LaMothe, Kimerer L. 2015. Why We Dance: A Philosophy of Bodily Becoming. Chicago: Columbia University Press.

McCormack, Derek P. 2014. Refrains for Moving Bodies: Experience and Experiment in Affective Spaces. Durham: Duke University Press.

Mcfee, Graham.1992. Understanding Dance. New York: Routledge. 
Mullis, Eric. 2015. "The Pragmatist Yogi: Ancient and Contemporary Yogic Somaesthetics." The Pluralist 10 (2): $205-$ 219. http://dx.doi.org/10.5406/pluralist.10.2.0205

Naharin, Ohad. 2006. "Truth in Movement." Dance Magazine 80 (10): 50-56.

Novack, Cynthia. 1990. Sharing the Dance: Contact Improvisation and American Culture. Madison: University of Wisconsin Press.

Protopapa, Efrosini. 2013. "Choreography as Philosophy, or Exercising Thought in Performance." In Thinking Through Dance: The Philosophy of Dance Performance Practices, edited by Jenny Bunker, Anna Pakes, and Bonnie Rowell, 118-135. Oxford: Dance Books.

Paxton, Steve. 1975. "Contact Improvisation." TDR: The Drama Review 19 (1): 40-42.

Schmeig, Anthony. 2004. Watching Your Back: Chinese Martial Arts and Traditional Medicine. Honolulu: University of Hawaii Press.

Sheets-Johnston, Maxine. 2015. The Phenomenology of Dance. Philadelphia: Temple University Press.

Singleton, Mark. 2010. Yoga Body: The Origins of Modern Posture Practice. Oxford: Oxford University Press.

Shusterman, Richard. 2012. Thinking Through the Body: Essays in Somaesthetics. Cambridge: Cambridge University Press.

___. 2008. Body Consciousness: A Theory of Mindfulness and Somaesthetics. Cambridge: Cambridge University Press.

_-_. 2000. Pragmatist Aesthetics: Living Beauty, Rethinking Art. New York: Rowman and Littlefield.

Summers-Bremner, Eluned. 2009. "Reading Irigaray, Dancing." Hypatia $15 \quad$ (1): 90-124. http://dx.doi.org/10.1111/j.1527-2001.2000.tb01081.x

Wartenberg, Thomas. 2006. "Beyond Mere Illustration: How Films can be Philosophy." Journal of Aesthetics and Art Criticism 64 (1): 19-32. http://dx.doi.org/10.1111/i.0021-8529.2006.00226.x

Young, Iris Marion. 1990. Throwing Like a Girl and Other Essays in Feminist Philosophy and Social Theory. Bloomington: Indiana University Press.

\section{Biography}

Eric Mullis is Assistant Professor of Philosophy at Queens University of Charlotte. He has recently published essays in Dance Research Journal, Journal of Aesthetic Education, and The Pluralist. He is currently an MFA student in dance at the University of Wisconsin (Milwaukee) and is a founding member of The Triptych Collective, a performing arts group based in Charlotte, North Carolina (USA). ericmullis8.com 